\title{
Gesture as a Cognitive Support to Solve Mathematical Problems
}

\author{
Mauro Francaviglia ${ }^{1}$, Rocco Servidio ${ }^{2}$ \\ ${ }^{1}$ Department of Mathematics, University of Turin, Turin, Italy; \\ ${ }^{2}$ Department of Linguistics, University of Calabria, Cosenza, Italy. \\ Email: mauro.francaviglia@unito.it, servidio@unical.it \\ Received October $7^{\text {th }}, 2010$; revised December $12^{\text {th }}, 2010$; accepted December $15^{\text {th }}, 2010$.
}

\begin{abstract}
The aim of this study was to investigate the relationships between gestures and mathematical problem solving. It concentrates on the idea that gestures can improve the student's mathematical conceptual abilities. The educational aim of the current study was to understand whether Penelope sewing the cloth every day will be eventually able to finish it in 50 days, before Ulysses returns in his home-town. To analyse children's gestures we applied the McNeill classification. The participants were five children aged between 9 and 10 years, attending the fifth-grade class of a primary school in Turin, Italy. We used the observational method to analyse the children's gestures behaviour. At the end of the analysis, we collected a corpus of 538 gestures. Results show that children use different gesture patterns to communicate their own mathematical ideas. Overall, these findings suggest that gestures facilitate children' learning of mathematical concepts and improve their cognitive strategies to the problem solution.
\end{abstract}

Keywords: Gestures, Mathematical Problem Solving, Mathematical Learning, Cognitive Processing

\section{Introduction}

In the last years many studies have attempted to better understand how the integration of gestures and spoken language can be used not only to improve the student's learning process of Mathematics means, but also to assess problem solving strategies (Alibali et al., 1999; Edwards, 2009; Radford, Edwards, \& Arzarello, 2009; Rasmussen, Stephan, \& Allen, 2004). Although some mathematical concepts are often difficult to understand for many students, a growing body of research shows how the semiotics perspective can be used to analyse the construction of mathematical concepts (Arzarello et al., 2009; Arzarello, Francaviglia, \& Servidio, 2006; Bazzini, 2001; Lim et al., 2009). Students are able to operate with gestures discovering how perceptual knowledge is a useful strategy to create into their own brain mental representations of mathematical problems.

When students work in the classroom, gestures are prevalent because they play an important role to support teaching and learning activities. Focusing on gestures role in the educational setting, researchers have examined the cognitive mechanisms of the gesture production as mental strategies that help children to reduce they cognitive effort when discusses about mathematical problems (Cook \& Goldin-Meadow, 2006; Goldin-Meadow et al., 2001).

The purpose of this study was to investigate how children use gestures to communicate mathematical ideas, improving the sharing of knowledge within the group. We consider the gestures as cognitive scaffolding that help children to solve mathematical problems. Another concurrent aim of this study was to contribute to the collection of a corpus of gestures within the domain of Mathematics. A conceptual framework on gestural mathematical behaviour represents an important chal- lenge to understand the role of non-verbal behaviour as a didactical strategy. In a typical cooperative work setting, gestural behaviours take the place of the images to communicate ideas about structured or abstract scientific topics.

To study the relationships between gestures and children's problem solving strategies, the Penelope's history has been selected as a didactical task for its appropriateness and appeal to the children's age. The problem was to understand whether Penelope sewing the cloth every day would be able to finish it in 50 day before Ulysses arrives. It is worthy that Penelope every day finishes a span of cloth, but during the night, she unpacks half of span. Knowing that the cloth must be 15 spans long; does Penelope manage to await the return of Ulysses without marrying? Finally, the interactions occurred while subjects worked to understand whether Penelope during her day/night work would be eventually able to complete successfully the spam before than Ulysses would come back to Ithaca. In this current research, we adopt the observational method to analyse children's gestural behaviour intent to solve mathematical problems. The observational method is a research technique suited to examine human and animal behaviour by investigating the body movements.

The paper is organized as follows. First, the theoretical background is briefly introduced. Next, Section 3 introduces and explains the research methodology of the current study. Results are described in the Section 4. Finally, in the last section conclusion and future direction that addressed the importance of the relationship between gestures and Mathematics are introduced.

\section{Theoretical Framework}

In the current work, the definition of gesture concerns the 
movements of the hands, in order to achieve some communicative aims performing meaningfully a didactical task. However, in the last years many broader definitions of gestures have been proposed. For an excellent overview of gesture research, see Kendon (2004).

Gestures not only support the mental processes but they are also used as mediators of a socio-cultural participation (Vygotskij, 1962). In this view, teachers and students share the same language offering a productive way to understand mathematical notions and then assuring them a good comprehension of the scientific knowledge. Researchers in communication and construction of mathematical meaning explored different ways to represent the scientific information. In particular, gestures are analysed not as an alternative approach to understand mathematical concepts, but rather as an integrative strategy, since they favour a natural communication mechanism able to easily deliver abstract ideas that are normally considered too complex to be understood. With respect to the gestures function they involve familiars' representational forms that can be used as didactical scaffolding during the mathematical problem solving, also improving the cognitive strategies of children.

The relationships between gestures and mathematical thinking have been analysed from different standpoints (Reynolds \& Reeve, 2002). Gestures and body movements have received great attention as means to investigate both mathematical thinking and communication strategies. This attention has increased since when Mathematics was considered as an embodied, socially and constructed human product (Bazzini, 2001). In particular, the aim of these studies was to investigate how gestures and bodily movements can influence the construction of mathematical meaning. The embodied vision of the gestures behaviour emphasize the importance to transform abstracts concepts in a visual and concrete form, making easier for people learning and using mathematical concepts during their educational activities (Gallese \& Lakoff, 2005; Nemirovsky \& Ferrara, 2009). Gestures have a direct role in the speech production process, where they facilitate the planning of it. When people speak, gestures play a role to organize conceptual ideas that are highly spatial or motoric (Kita, 2003). Other studies have shown that gestures are more linked with words that are spatial and concrete than with words that are non-spatial and abstract (Goldin-Meadow, 2003; Lakoff \& Núñez, 2000).

As well as based on this approach, gestures are a particular modality of embodied cognition; they are connected to the speech and they can serve as important bridges between internal imagery (or mental state) formal and symbolic expressions of mathematical ideas (Bazzini, 2001; Kita, 2000). Gestures are therefore an important source of information, since body movements support the oral communication reducing language ambiguity and improving the share of scientific concepts. In addition, gestures are related to problem solving process reinforcing and improving the conceptual representation of mathematical concepts. When all subjects share within the group the same cognitive strategies, this mechanism reduces their mental effort necessary to apply the problem solving methodologies.

\section{Methodology}

\section{Participants}

The participants were selected from an age interval within which previous studies had provided different examples of how children use gestures to solve mathematical problems (Arzarello et al., 2006; Cook \& Goldin-Meadow, 2006). The participants were five subjects ( 2 males and 3 females), aged between 9 and 10 years $(M=9.8 ; S D=0.35)$, attending the fifth-grade class of a primary school in Turin, Italy. For the purpose of this study, the students remained in their classroom, and then worked in-group to solve the mathematical problem. No preliminary test was used to measure the participant's cognitive abilities. They participated to the research after having obtained their consent from the school Director.

\section{Procedure}

Participants worked in their school to grant them a familiar environment. Each work session lasted approximately 40 minutes. The session began with the reading by children of their task. While the participants worked to solve the problem, the researchers remained in the classroom and videotaped their activity. However, researchers did not give to participants any tip or suggestion but just supported them by providing paper and other materials. Using a digital video camera, which was situated on a tripod during all the observational sessions, we videotaped the participant behaviours. The video camera was set, in order to record carefully all participants' activities and making easier the next codifier work.

After this initial phase, the second step was to analyse the data analyze by applying the observational method. We recruited one codifier with the intent to codify participant gestures. The codifier, before starting to collect the behavioural data, followed a training course on how to recognize the behaviours (or gestures) listed in the taxonomy. Thus, the codifiers with the support of a researcher better learn in fact to recognize all behaviours and after this training session the codifiers began with the behavioural data.

\section{Materials}

The gestures were codified from the videotapes among participants' interactions in the school, using the taxonomy designed according to the previous studies (Edwards, 2009; Radford, Edwards, \& Arzarello, 2009). The taxonomy has been designed by observing the participants behaviour while they worked to solve the mathematical problem. The taxonomy aim was twofold. The first aim was to record the behavioural gestures linked with the mathematical concepts; the second aim was to collect the behavioural actions that participants realized during the workgroup session.

Then, we accomplished a taxonomy that included five main gestures macro-categories: Deictic Gestures; Iconic Gestures; Regulator Gestures; Adaptive Gestures; Problem Solving Gestures. In order to define the participant's activity, we have individuated for each behavioural macro-categories other specific micro-behaviours.

The gestures were initially identified by using the scheme of McNeill's (1996) classification. After this initial work, we have individuated further categories. The most important gesture types individuated by McNeill (1996) are: Iconic Gestures, which "bear a close formal relationship to the semantic content of speech". Metaphoric Gestures means, "the pictorial content presents an abstract idea rather than a concrete object or event" (p. 14). Beat, a repetitive gesture that "indexes the word or 
phrase it accompanies as being significant" (p. 15), and Deixis, a "pointing movement [that] selects a part of the gesture space" (p. 80). The gestures produced during the participant's activities were analysed by using the observational method. Table 1 shows an example of the gestures taxonomy used to analyse participant's behaviour.

\section{Defining the Target Behaviour}

The target gestures (or taxonomy) were the result of the participant's behavioural analysis while they were intent to solve the mathematical problem. As we mentioned above, the researcher analysing participant's behaviour has identified and analysed five main behavioural categories:

1) Deictic Gestures: we intend behaviours in which the subject uses them to point and serve to move the listener attention from the speaker to the near region of the referent. Deictic Gestures indicate also how to individuate things, directing-to and positioning-for. These behaviours are used to determine how the subjects interact not only with objects but also communicate ideas and conceptual aspects by gestures.

2) Iconic Gestures: all gestures used to illustrate spoken words. Subjects use them to encode difficult messages, reducing the communicative effort of the speaker and then helping the receiver to easily decode the messages. As a typical example, illustrators emphasize actions that represent geometric shapes. Among these behaviours, we include actions that illustrate certain aspects that connect spoken language and gestures. In particular, these gestures provide emphasis to concurrent speech, controlling the conversation between subjects.

3) Regulators Gestures: all the gestures that support both interaction and communication between emitting and recipient. These behaviours control the flow of conversation such as nodding the head up and down to indicate agreement and as though signalling the other to continue the conversation.

4) Adaptors Gestures: all the gestures that are not used intentionally during the communication or interaction. These behaviours include actions that presuppose the subject's ability to equilibrate their own motivational internal state to continue their activity. They include behaviours in which subjects touch some body parts as cognitive strategies to improve the quality of the conceptual analysis of the problem.

5) Problem Solving Gestures: we intended all gestures used by subjects to help other colleagues while work together to solve mathematical problems. In particular, we intend all behaviours that indicate the cognitive strategies that subject realize to solve the problem.

\section{Reliability}

A student coder, blinded to the main study hypothesis, was trained both to use the taxonomy and to apply the rating system methodology. The coder analysed several examples of the videotapes collected during the participant's problem solving activities. At the end of each training session, we checked the coder performance by calculating an inter-rate agreement. We compute the percent agreement between coder and researcher involved in the current study. According to the taxonomy behaviour, we have checked the rate achieved to identify correctly the participants' gestural behaviour. To determine the inter-reliability during data coding, we analysed randomly some participant's behaviour selected from collected videotape. The
Table 1.

Example of regulators gestures.

\begin{tabular}{ll}
\hline \multicolumn{1}{c}{ Code } & \multicolumn{1}{c}{ Description } \\
\hline Head movements & Children move the head, neck, eyes \\
Hand gestures & Children use hands to touch some body parts \\
Posture & Body postures and other parts such as torso and arms \\
\hline
\end{tabular}

inter-reliability was calculated by using the Cohen Kappa statistic method, which agreement index obtained ranged from 0.70 to 0.80 . Generally, a Kappa value of 0.70 is considered to be satisfactory.

\section{Coding Gestures}

Each observational session was analysed for every behavioural category, for each participant of all groups and for the whole duration of the work session. We have adopted the direct observation method to analyse participant behaviour. The coder observed a single participant for the entire time, recording the beginning and the end of each behavioural pattern (duration) of all instances of the taxonomy behaviours. Besides, the coder counted each single duration pattern obtaining the frequencies number of the behaviours realized by participants. We have used a checklist method to register participant's behaviour, which is an inventory of all target behaviours that the codifier marked when the behaviour occurred.

The videotape analysis was segmented into meaningful action units. In fact, we coded only behaviours in which the participant performed gestures directed to solve the mathematical problem. For example, moving in the room was not considered as a relevant behaviour for our analysis, whereas asking the support or explaining to other participants' own ideas how to solve the problem was instead coded as a relevant behaviour. Both the frequencies of the relevant actions and the duration they lasted were registered.

\section{Statistical Analysis}

We have used the chi-square statistical test to evaluate the relationships between frequency and duration in comparison with each gesture category. We needed to determine whether the distribution of gesture behaviour for frequency was significantly different from the distribution of the duration.

\section{Results}

\section{Quantitative Summary}

A corpus of 538 gestures was collected analysing the videotape session; there was a total of 20 minutes of videotape data. The corpus does include a large number of Deictic Gestures (e.g., use of the paper and other behaviours) that occurred while the participants worked together to solve a written problem. The participant's gestures were produced while they discussed to find the problem solution. The average rate of gestures production while the participants discussed within the group was 3.32 gestures per minute. The total gestures produced by each participant during the problem solving session were 39.03 . Table 2 shows the major gestures realized by participants during the problem solving session. 
Table 2 .

Types of gestures by most salient dimensions.

\begin{tabular}{lcccccc}
\hline & Deictic & Iconic & Regulator & Adaptive & $\begin{array}{c}\text { Problem } \\
\text { solving }\end{array}$ & Total \\
\hline Frequency & 74 & 72 & 139 & 109 & 144 & 538 \\
Percentage & 13.75 & 13.38 & 25.84 & 20.26 & 26.77 & 100 \\
\hline
\end{tabular}

The participant gestural behaviours have also been analysed. Chi-square result showed no significant differences among gestures behaviour and the two variables measured (frequency $\chi^{2}(19)=4.60, p>.05$ and duration $\left.\chi^{2}(17)=7.40, p>.05\right)$. Statistical analysis revealed that gestural behaviours were distributed among all participants' activities. For the educational task proposed, we found that participants realized several gestures that evolved during the different phases of the whole discussion process. When the participants' comprehension of the problem was better, then them realized more articulated gesture configurations. This result confirms that gestures are important for all phases of the problem solving process, and then was associated with specific behavioural category. In the next section, we give an overview of the principal gestures behaviours that participants realized during their educational activities.

\section{Qualitative Summary}

In this section, we provide a qualitative analysis of the participants' gestural behaviour. They realized different type's gestures to communicate their own ideas to other people, in order to infer the solution of the problem. The gestures supported the discussion among all participants; in particular, when they are not yet able to individuate the correct solution of the problem. We analysed the participants' gestural behaviour while they worked together to find the solution.

As we already said, the problem assigned was to understand if Penelope sewing the cloth every day would be able to finish it in 50 day before Ulysses arrives. To solve this problem, all participants realized gestures and used structured objects such as paper and pencil to represent their own concepts. Participants needed to combine gestures and tools reinforcing their mental representation of the mathematical concepts and then to use them to explain and describe the problem.

The gestures shown in Figure 1(a) and in Figure 1(b) represent two kinds of iconic movements, which refer to concrete actions or events (Edwards, 2009). Both participants described an imaginary dimension of the span, displaying clearly the physical process regarding the construction of it. After, see Figure 1(b), the hand movement is similar, but they realized a shape that includes a spatial dimension of the span. In virtue of their shared socio-cultural background, participant interpreted easily these gestures as referring to the action, which they mean to represent the dimension of a span.

When gestures and speech are correctly associated, subjects reproduced the same hand movement confirming their active role of the attention in the learning process (see Figure 2). Participants used behaviours compatible with their verbal language and relied on paper writing to make more comprehensible their mental representation of the concepts. Gestures reproduced by other people represent thence a careful cognitive support to stimulate the discussion within the group, improving the quality of communication.

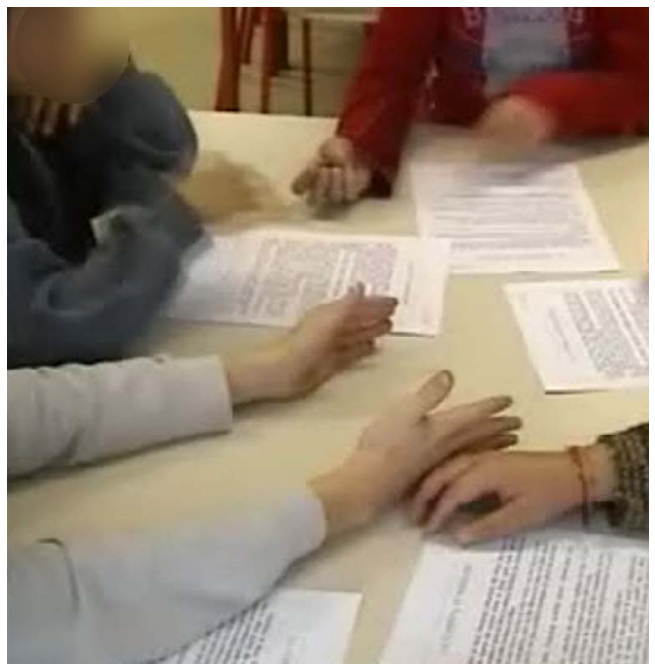

(a)

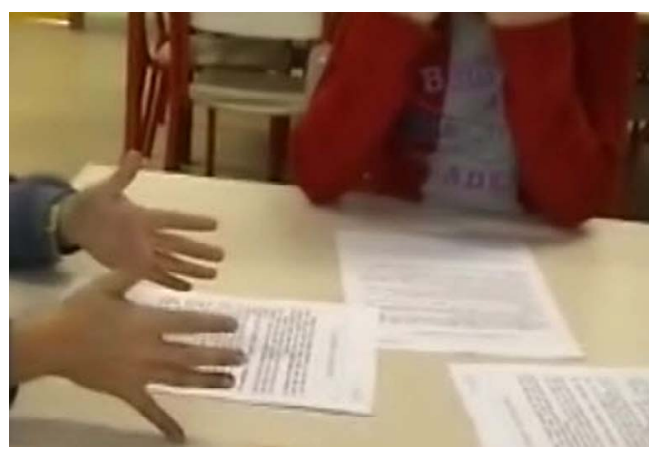

(b)

Figure 1.

Hand gesture that represents precise shape.

In Figure 3(a) and Figure 3(b) subjects combine a conceptual integration between gestures and tools. In this context, the affordances of the gesture hand and the physical objects allow participant to produce a new conceptual analysis of the problem solving. Participants, in turn, analysed the mathematical procedure by creating a new shape or conceptual strategy to identify the correct solution of the problem under investigation.

We needed to determine whether the distribution of gesture behaviour for frequency was significantly different from the distribution of the duration.

\section{Discussion}

Examining participant's behaviour, we found that gestures reinforced the scientific communication (e.g., gestures and speech converged on the same hand representation), so they tried to find a solution bearing in mind the hand representation. When the gestures were more structured and correct, participants reduced their spontaneous production focusing on specific aspects of the problem. In addition, these representations improved the spatial relationship between speech and hand movement as showed in Figure 1(a) and Figure 1(b). In fact, the form of the gestures with palms perpendicular to the table and facing each other, suggested that children attempted to realize a 


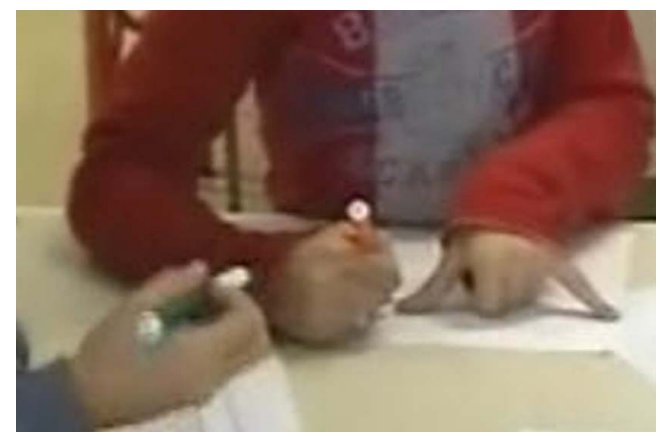

(a)

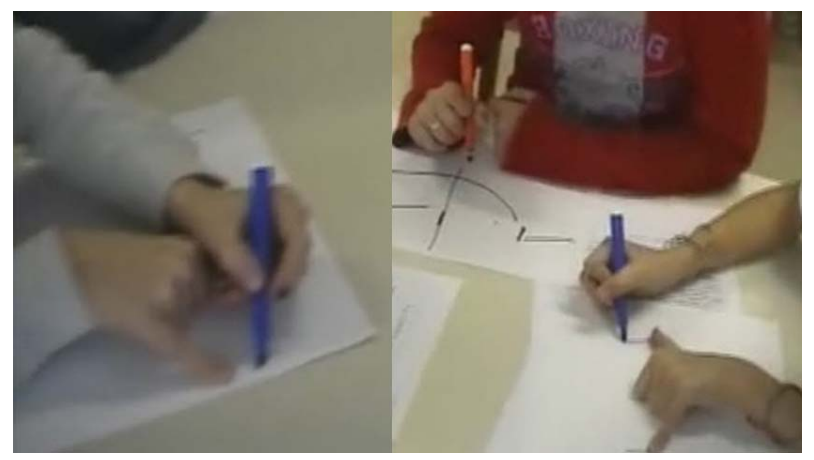

(b)

(c)

Figure 2.

Subject (a) reproduces the initial shape. Other subjects reproduce the same shape $((b)$ and $(c))$.

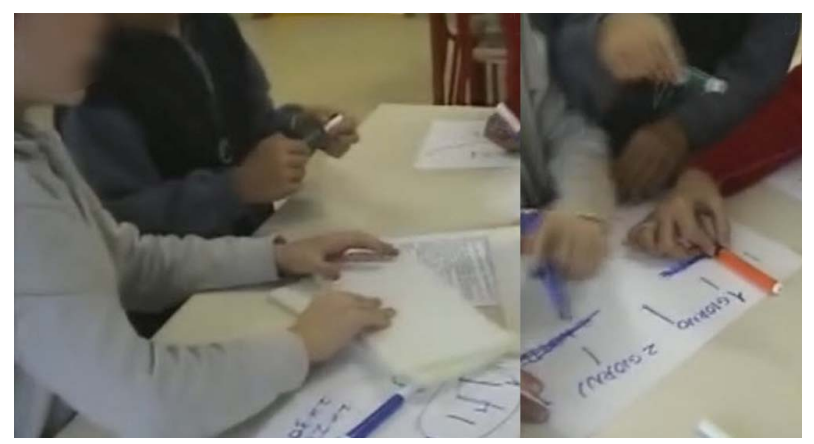

(a)

(b)

Figure 3.

Objects and tools as support solving mathematical problems.

mental model of the span conceptualizing it and therefore reducing its abstract meaning (Arzarello et al., 2009; Arzarello et al., 2006; Bazzini, 2001). Therefore, these gestures represent also a metaphoric idea of the concept of span; ideal entities are treated as objects. Participants tried to understand the span properties represented it by using hand movements. These behaviours provided to the participants concrete entities that allow them to express themselves through the gestures abstract means.

Based on obtained results, we propose a conceptual scheme of gesture behaviour, which aim is to outline the gesture/perception cycle as a dynamic mechanism that their production involves (Figure 4). Initially, the subjects produced a gesture that modifies the environmental context, by creating new stimuli. After, the subjects perceived this behavioural pattern and then analysed it, in order to identify the conceptual properties that gestures communicate.

An important aspect of the analysis phase concerns the conceptualization process. In this phase, subjects plan the next movement, showing how the cognitive processes elaborate the problem aims and then they decide the action that it is more suitable, in order to achieve the correct solution or to revise the previous mental strategies.

According to Parrill and Sweetser (2004) even metaphoric gestures have an iconic property, since by means of the hand shapes and movement, they appeal to some visual or concrete representation. These situations offer specific elements that underlie an inferential thinking as support to the children understanding of the abstract concepts expressed by using gestures movements. Specifically, a metaphoric gesture includes two conceptual mapping sequences: one iconic between real space and visual/concrete situation, and a second one between the conceptual space and the intended abstract meaning (Edwards, 2009). Gestures allow the subjects to manipulate mathematical concepts by using only certain kind of actions and configurations, which offer concrete and stable representations of the abstract concepts. These kinds of gestures display some type of iconicity. We can see how the gestures refer to a symbolic content rather than to a concrete object. This type of reference is called "chain of signification" (Edwards, 2009).

Finally, gestures can be seen as simple illustrations of students' ideas, which incorporated hands-on materials into conceptual mathematical aspects (see Figures 2(a), 2(b), 2(c)). Gestures and tools may play an important role in students' learning. In this context, the interaction between gestures and tools acted as scaffold to support the development of a conceptual understanding. This process encouraged the discussion and other appropriate cognitive strategies that supported the subjects thinking. Besides, these are clear examples that a more efficient cognitive strategy requires representational forms to communicate own conceptual ideas.

\section{Conclusion}

In this paper, we have examined the role of gestures as facilitators to solve mathematical problems. We have argued that gestures play an important role for the learning and that they assist children in acquiring, restructuring and tuning their own knowledge. Moreover, much of learner's conceptual knowledge is socially constructed and shared, and gestures should be used to assist them in this endeavour. Our findings show that gestures reveal important information about children's reasoning on mathematical problem solving strategy. Children realize

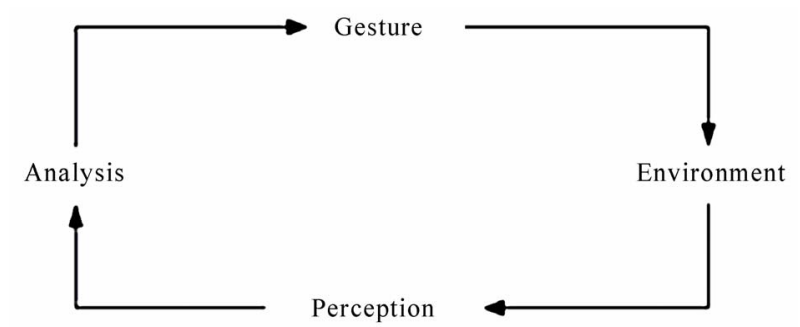

Figure 4.

Conceptual scheme to represent the gesture/perception cycle. 
different kinds of gestures that demonstrate how the problem solving processes include behavioural categories that enhance the conceptualization phase. More specifically, gesture provides a more complete representation of the problem and then of the solution rather than just speech alone. In addition, in this paper we have also identified the importance to use tools, which not only support children's reflection, but also provide a very powerful predictor of the problem solution.

These gestures are an evidence of how children think about mathematical concepts, once they have learned how to manipulate them symbolically. All children involved in the current investigation produced gestures that evoked hands-on activities, such as drawing and physical manipulation of the ideal structures. The drawing activities represent a further analysis of the quality of gesture structure. In fact, many of these gestures were precise with regard to the structural details of the agreement cloth structure. Our findings are in line with other studies that have investigated the role of gestural behaviour from an educational standpoint (Church, Ayman-Nolley, \& Alibali, 2001; Goldin-Meadow, 1999; Kelly et al., 2002; Singer \& GoldinMeadow, 2005). In addition, these studies are significant also from an educational perspective since they represent a new analytic framework to analyse the children cognitive process involved in learning and in problem solving activities. In particular, gestures can be successfully used in the didactical context as a natural support without modifying the natural children behaviour.

We have also shown here that gestures evolve during the discussion process. Initially, children produce gesture that is more spontaneous. In this phase, the children's ideas about the problem are not always clear. After this initial phase, children increased their problem understanding by incorporating new information and then creating an articulated mental representation of the assigned problem. This conceptualization was expressed by using gestural movements. Therefore, understanding how children represent problems will allow to better explaining how they construct and identify their cognitive strategies to solve a mathematical problem. Furthermore, this connection between gestures, speech and drawing makes the children more aware of the cognitive strategies available that they can apply to solve a mathematical problem. For example, children sometimes represented the length of the span by using some gestures, showing how the problem entities changed continuously. Such cases represent important aspects that show how children's thinking evolves during the problem solving activities. During these didactical activities, children construct in fact new strategies (not always correct) that often modify other realized previously.

\section{Further Direction}

Our research provides a large scientific evidence of the relationship existing between gestures and mathematical problem solving. A further investigation should be to examine also the relationship between gesture and metacognition (Perfect \& Schwartz, 2002). Metacognition encompasses a set of procedures that allow cognitive systems equipped with it to predict or evaluate their ability to perform a given cognitive operation. This problem is being currently addressed and will be the subject of forthcoming publication. It will allow to individuate, for example, a possible distinction between cognitive and meta- cognitive gestures.

\section{Acknowledgements}

We warmly thank Prof. Ferdinando Arzarello head of the Department of Mathematics of University of Torino, Italy, for having provided the educational material (video) on children activities. We also thank the student (Eleonora Crudo) of the University of Calabria (Cosenza, Italy) that has analysed the children behaviours.

\section{References}

Alibali, M. W., Bassok, M., Solomon, K. O. S., Syc, E., \& GoldinMeadow, S. (1999). Illuminating mental representations through speech and gesture. Psychological Science, 10, 327-333. doi:10.1111/1467-9280.00163

Arzarello, F., Bazzini, L., Ferrara, F., Robutti, O., Sabena, C., \& Villa, B. (2006). Will Penelope choose another bridegroom? Looking for an answer through signs. In J. Novotná, H. Moraová, M. Krátká, \& N. Stehlíková (Eds.), Proceedings of the 30th Conference of the International Group for the Psychology of Mathematics Education (Vol. 2, pp. 73-80). Prague: Charles University.

Arzarello, F., Francaviglia, M., \& Servidio, R. (2006). Gesture and body-tactile experience in the learning of mathematical concepts. In Proceedings of the International Conference on Applied Mathematics-APLIMAT (pp. 253-259). Bratislava, SL: University of Slovenia.

Arzarello, F., Paola, D., Robutti, O., \& Sabena, C. (2009). Gestures as semiotic resources in the mathematics classroom. Educational Studies in Mathematics, 70, 97-109. doi:10.1007/s10649-008-9163-z

Bazzini, L. (2001). From grounding metaphors to technological devices: A call for legitimacy in school mathematics. Educational Studies in Mathematics, 47, 259-271. doi:10.1023/A:1015143318759

Church, R. B., Ayman-Nolley, S., \& Alibali, M. W. (2001). Crossmodal representation and deep learning. Annual meeting of the cognitive development society. Virginia Beach, VA.

Cook, S. W., \& Goldin-Meadow, S. (2006). The role of gesture in learning: Do children use their hands to change their minds? Journal of Cognition and Development, 7, 211-232. doi: $10.1207 / \mathrm{s} 15327647 \mathrm{jcd} 07024$

Edwards, L. (2009). Gesture, conceptual integration and mathematical talk. International Journal for Studies in Mathematics Education, 1, 33-46.

Gallese, V., \& Lakoff, G. (2005). The brain's concepts: The role of the sensory-motor system in conceptual knowledge. Cognitive Neuropsychology, 22, 455-479. doi:10.1080/02643290442000310

Goldin-Meadow, S. (1999). The role of gesture in communication and thinking. Trends in Cognitive Sciences, 3, 419-429. doi:10.1016/S1364-6613(99)01397-2

Goldin-Meadow, S. (2003). Hearing gestures: How our hands help us think. Chicago: University Press.

Goldin-Meadow, S., Nusbaum, H., Kelly, S. D., \& Wagner, S. (2001). Explaining math: Gesturing lightens the load. Psychological Science, 12, 516-522.doi:10.1111/1467-9280.00395

Kelly, S. D., Singer, M., Hicks, M. J. \& Goldin-Meadow, S. (2002). A helping hand in assessing children's knowledge: Instructing adults to attend to gesture. Cognition and Instruction, 20, pp. 1-26. doi:10.1207/S1532690XCI2001 1

Kendon, A. (2004). Gesture: Visible action as utterance. Cambridge, UK: University Press.

Kita, S. (2000). How representational gestures help speaking. In D. McNeill (Ed.), Language and gesture (pp. 162-185). Cambridge: Cambridge University Press. doi:10.1017/CBO9780511620850.011

Kita, S. (2003). Pointing: A foundational building block of human communication. In S. Kita (Ed.), Pointing: Where language, culture and cognition meet (pp. 1-9). Mahwah: Lawrence Erlbaum. 
Lakoff, G., \& Núñez, R. (2000). Where mathematics comes from: How the embodied mind brings mathematics into being. New York, NY: Basic Books.

Lim, K. V., Wilson, J. A., Hamm, J. P., Phillips, N., Iwabuchi, J. S., Corballis, C. M., Arzarello, F. \& Thomas O. M. (2009). Semantic processing of mathematical gestures. Brain and Cognition, 71, 306312. doi:10.1016/j.bandc.2009.07.004

McNeill, D. (1996). Hand and mind: What gestures reveal about thought. Chicago: The University of Chicago Press.

Nemirovsky, R., \& Ferrara, F. (2009). Mathematical imagination and embodied cognition. Educational Studies in Mathematics, 70, 159174. doi: $10.1007 / \mathrm{s} 10649-008-9150-4$

Parrill, F., \& Sweetser, E. (2004). What we mean by meaning. Gesture, 4, 197-219. doi:10.1075/gest.4.2.05par

Perfect, T. J., \& Schwartz, B. L. (2002). Applied metacognition. Cambridge: Cambridge University Press.
doi:10.1017/CBO9780511489976

Radford, L., Edwards, L., \& Arzarello, F. (2009). Introduction: Beyond words. Educational Studies in Mathematics, 70, 91-95. doi:10.1007/s10649-008-9172-y

Rasmussen, C., Stephan, M., \& Allen, K. (2004). Classroom mathematical practices and gesturing. Journal of Mathematical Behavior, 23, 301-323. doi:10.1016/i.jmathb.2004.06.003

Reynolds, F. J., \& Reeve, R. A. (2002). Gesture in collaborative mathematics problem-solving. Journal of Mathematical Behavior, 20, 447-460. doi:10.1016/S0732-3123(02)00091-3

Singer, M. A., \& Goldin-Meadow, S. (2005). Children learn when their teacher's gestures and speech differ. Psychological Science, 16, 8589. doi:10.1111/i.0956-7976.2005.00786.x

Vygotskij, L. S. (1962). Thought and language. Chicago: MIT Press. doi:10.1037/11193-000 